\title{
Modelling and static stability analyses of the hexa-quad bimorph walking robot
}

\author{
Dominik Wojtkowiak ${ }^{1, *}$, Krzysztof Talaśka ${ }^{1}$, Ireneusz Malujda ${ }^{1}$, Jan Górecki ${ }^{1}$ and \\ Dominik Wilczyński ${ }^{1}$ \\ ${ }^{1}$ Chair of Basics of Machine Design, Poznan University of Technology, ul. Piotrowo 3, 60-965 \\ Poznań, Poland;
}

\begin{abstract}
Gait stability of the walking robot has great influence on the many functional features related to walking robots, from mechanical construction to control algorithms and generating steps. The goal of the presented research was a development of the concept of hexa-quad bimorph walking robot. Results of the performed static stability analysis allowed the initial verification of the mathematical model and provided information about the design adequacy and the possibilities of controlling the machine. The research involved analysing the characteristic postures of the robot focused on retaining static stability. To achieve this objective the mathematical model was made to determine the centre of gravity for the robot by using Denavit-Hartenberg notation. On this basis the simulation model was created in Matlab Simulink environment, where the described analyses were conducted. Based on the obtained results, the initial model error was determined at approximately $3 \%$. It was also established that the centre of gravity for the design was not significantly different from the effective centre of gravity for the robot. This made it possible to achieve static stability through adequate alignment of the legs in nearly every robot configuration.
\end{abstract}

Keywords: walking robot, hexa-quad, bimorph, hexapod, quadruped

\section{Introduction}

Gait stability is one of the most important parameter, which has an influence on a number of functional features related to walking robots, from mechanical construction to control algorithms and generating steps. For walking robots we can distinguish two types of stability: static stability and dynamic stability. The measurement value used to determine stability margin can be either distance, angle, energy, moment or force. On their basis many stability criteria have been formulated. The same methods can be used to determine both types of stability by making appropriate assumptions. One example is the energy stability margin which involves measuring the difference between the actual potential energy and the initial one. Considering that all external forces including the force of gravity act on the centre of

\footnotetext{
${ }^{*}$ Corresponding author: dominik.wojtkowiak@put.poznan.pl

Reviewers: Andrzej Kołodziej, Ján Vavro
} 
gravity of the robot the dynamic energy stability margin may be obtained [1, 2].

In this paper authors focused solely on static stability. The main assumption of static stability of walking robots is retaining centre of gravity or its projection inside the support polygon. Support polygon is defined as a two-dimensional figure created from coordinates of tips of all supporting legs projected onto a horizontal plane. As a quantitative criterion static stability margin (SSM) and longitudinal static stability margin (LSM) were used Figure 1. Longitudinal static stability margin is defined as the shorter one out of the distances between the projection of centre of gravity on the horizontal plane and the edge of the support polygon as measured along the direction of the walking robot's speed vector. In turn, static stability margin is defined as the shortest distance between the aforementioned projection and the edge of the support polygon. [3, 4] These methods can be only used on in cases of negligible inertia and absence of forces, which counteract the robot's movement. However, irregularities of the surface where the robot is moving around do not have influence on adequacy of results obtained by means of one of the above mentioned methods [5].

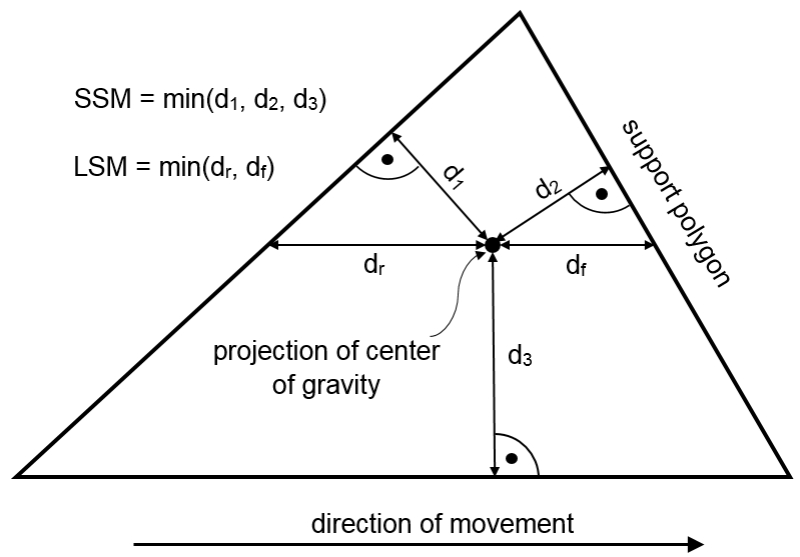

Fig. 1. Support polygon together with indicated stability margins [1]

This paper focuses on considerations of how important the placement of the centre of gravity of the hexa-quad bimorph walking robot is with a view to its static stability (section 2 ). The objective of research presented in section 3 is drawing important conclusions regarding both the design adequacy and motion control methods. It also allows potential modification and initial verification of the universal mathematical model of the hexa-quad bimorph walking robot.

Hexa-quad bimorph walking robot has an unique ability to move easily from six-legged posture to four-legged posture or the other way around. The key of this transformation is to achieve the highest possible speed while moving through uneven terrain combined with an ability to manipulate objects while robot is in motion or at a standstill. Each of this function can be fulfilled during remaining in one of the configurations - locomotion is realized in HEXAPOD configuration (Fig. 2a), while object manipulation can be made during QUADRUPED phase (Fig. 2b). From the existing walking robot construction, we can distinguish few, that can fulfil above mentioned features [6-10], but none of them can fit into both descriptions.

Basically hexa-quad bimorph walking robot consist of three-segment body, where each segment is assigned only by one pair of limbs, which are either locomotive or manipulative and locomotive legs (the front ones). Between front and middle segment of the body active double axial joint is located, while between middle and rear body trunks is only single axial joint. Thanks to this solution, configurations can be changed by tilting up the front body 
segment. The concept and the construction of the hexa-quad bimorph walking robot was precisely described in authors previous papers $[1,11,12]$.

a)

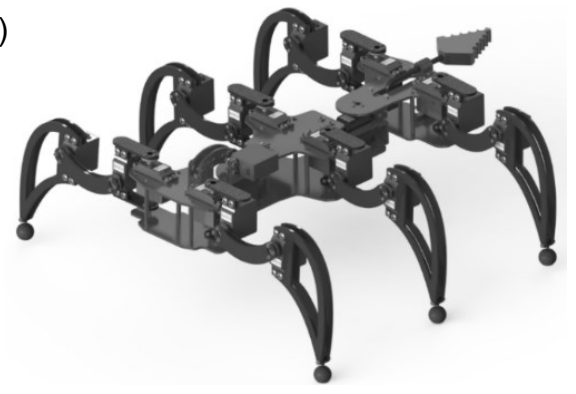

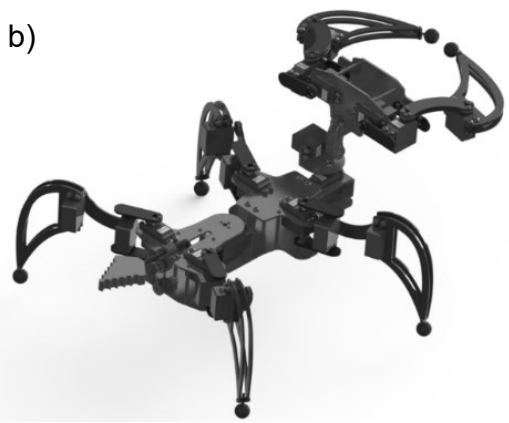

Fig. 2. 3D model of the hexa-quad bimorph walking robot [1] a) in HEXAPOD configuration, b) in QUADRUPED configuration

\section{Mathematical model of the hexa-quad bimorph walking robot's centre of gravity}

Analytically formulated mathematical model of the centre of gravity and the simulation model formulated on its basis provide a lot of information about the design of the walking robot. On its basis it is possible to perform both analyses of static stability and basic tests verifying the adequacy of the design with a view to weight distribution, motion capabilities and assumed control algorithms. In this paper authors presents static stability analyses, which was made based on the model formulated with using methodology presented in [12] and following assumptions.

\subsection{Basic assumptions used in modelling}

The basic assumption needed in order to determine the walking robot's centre of gravity is establishing coordinate systems used during analytical formulations. Coordinate systems used in the described methodology are presented in Figure 3. All presented coordinate systems, except the system $(\mathrm{KS})_{0}$ and $(\mathrm{KS})_{\mathrm{s}}$, are located on the common plane XY.

In the assumed methodology of determining he centre of gravity, the walking robot is divided into the following segments: front body (KP) together with manipulation and locomotion legs (NP1 and NL1), rear body (KT) together with locomotion legs (NP3 and NL3), middle body (KM), right locomotion leg (NP2) and left locomotion leg (NL2). The result of the calculations, conducted according to the described methodology, is the position of the walking robots centre of gravity relative to the base coordinate system. This means that the methodology does not take into account the robot's tilt relative to the surface and the weight it is elevating.

At all stages of formulating the mathematical model of the walking robot geometric methods, Denavit-Hartenberg notation and basic transformation matrices (rotation and translation) [12] have been used. Taking into account the assumptions and the obtained results, the range of generalized coordinate changes for the walking robot's legs is presented in Table 1. 


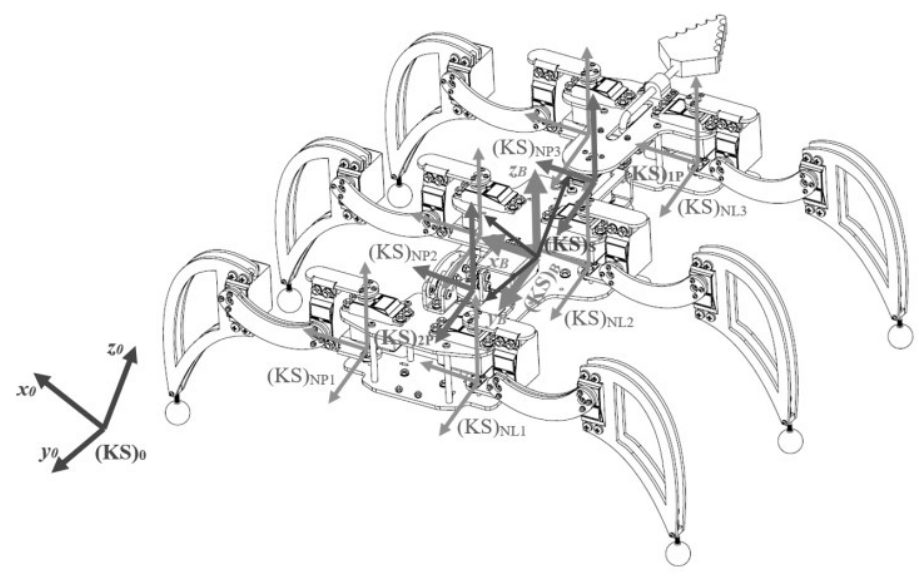

Fig. 3. Indicating coordinate systems of the hexa-quad bimorph walking robot [12]

Table 1. Range of changes of the walking robot's generalized coordinates

\begin{tabular}{|c|c|}
\hline For the right leg: & For the left leg: \\
\hline$\theta_{1_{N P i}}=-90^{\circ}$ to $90^{\circ}$ & $\theta_{1_{N L i}}=-270^{\circ}$ to $-90^{\circ}$ \\
\hline$\theta_{2_{N P i}}=-90^{\circ}$ to $90^{\circ}$ & $\theta_{2_{N L i}}=-90^{\circ}$ to $90^{\circ}$ \\
\hline$\theta_{3_{N P i}}=-180^{\circ}$ to $0^{\circ}$ & $\theta_{3_{N L i}}=0$ to $180^{\circ}$ \\
\hline
\end{tabular}

\subsection{Simple kinematics of walking robot leg}

To draw the support polygon we need the coordinates of every foot of the robot. In order to do that it is necessary to solve the equation of simple kinematics and then perform the appropriate transformations to adapt the obtained model to each leg. The same methods were used for simple kinematics as for determining the position of the leg's centre of gravity. Using values indicated in the Table 1 and the lengths of three segments of the leg $L_{1}, L_{2}, L_{3}$, the following mathematical model emerges:

$$
\left[\begin{array}{c}
x_{N P i} \\
y_{N P i} \\
z_{N P i}
\end{array}\right]=\left[\begin{array}{c}
L_{1} c_{1}+L_{2} \cdot c_{1} \cdot c_{2}+L_{3} \cdot c_{1} \cdot c_{23}+a_{4} \cdot c_{1} \cdot c_{235}-y^{\prime \prime}{ }_{N P i} \cdot s_{1} \\
L_{1} s_{1}+L_{2} \cdot s_{1} \cdot c_{2}+L_{3} \cdot s_{1} \cdot c_{23}+a_{4} \cdot s_{1} \cdot c_{235}+y^{\prime \prime}{ }_{N P i} \cdot c_{1} \\
L_{2} \cdot s_{2}+L_{3} \cdot s_{23}+a_{4} \cdot s_{235}
\end{array}\right],
$$

where: $c_{23}=\cos \left(\theta_{2}+\theta_{3}\right), s_{23}=\sin \left(\theta_{2}+\theta_{3}\right)$,

$$
c_{235}=\cos \left(\theta_{2}+\theta_{3}+\theta_{5}\right), s_{235}=\sin \left(\theta_{2}+\theta_{3}+\theta_{5}\right) \text {. }
$$

Transformations for the left leg are performed according to the assumptions described in subsection 2.1. In order to use the obtained coordinates they need to be expressed relative to the coordinate system $(\mathrm{KS})_{\mathrm{B}}$. This kind of transformation can be performed analogously to the calculations described in the methodology of determining centre of gravity of the robot's rear and front body segments. The same matrices of translation and rotation have been used. The obtained coordinates are used to draw the support polygon, which is used to determine static stability margin. 


\section{Simulation analyses of the hexa-quad bimorph walking robot's static stability}

\subsection{Methodology}

Based on the mathematical model of the hexa-quad bimorph walking robot the simulation model was formulated in the computer environment of Matlab Simulink. Fragment of the recreated model (subsystem) related to the position of centre of gravity of the thigh segment of the right leg NP1 is presented in Figure 4.

This model uses basic blocks such as constant, gain, product, sum, divide, scope, input, output, sine, cosine, etc. Due to the model's complexity it was divided into multiple subsystems. In the presented model there are two ways of assigning generalized coordinates. The first method, used to analyze particular postures of the robot, is assigning constant values. This method was used in tests whose results are presented in this article. The alternative method is testing full walks of the robot simulating the settings of the runs on appropriate Signal Builder blocks. This method requires much more complicated procedures to analyze the obtained results including discretization of measurements with a given time step.

Research described in this article involved the analysis of the hexa-quad bimorph walking robot's characteristic postures and establishing their static stability in a given configuration. To analyze stability the quantitative and the qualitative criteria have been used. The research objective is mostly to initially verify the formulated mathematical model. Additionally, they carry information about the adequacy of the robot's design, motion control as well as its locomotive capabilities and the machine's limitations. They are also a source of reference for future tests to be performed on the physical object. The results are presented in the form of graphs showing the XY plane, where the position of all the robot's feet is indicated, the support polygon and its centre of gravity according to the legend included in Table 2. The qualitative criterion of static stability is the position of centre of gravity inside or outside the support polygon. Static stability margin (SSM) and longitudinal stability margin (LSM) are used as the quantitative criterion.

Table 2. Legend to the graphs presenting testing results

\begin{tabular}{|c|c|}
\hline Symbol & Meaning \\
\hline & $\begin{array}{c}\text { position of the tip of the foot currently in the retraction (support) } \\
\text { phase }\end{array}$ \\
\hline $\mathrm{O}$ & $\begin{array}{l}\text { position of the tip of the foot currently in the protraction } \\
\text { (transportation) phase }\end{array}$ \\
\hline$x$ & the walking robot's centre of mass \\
\hline
\end{tabular}




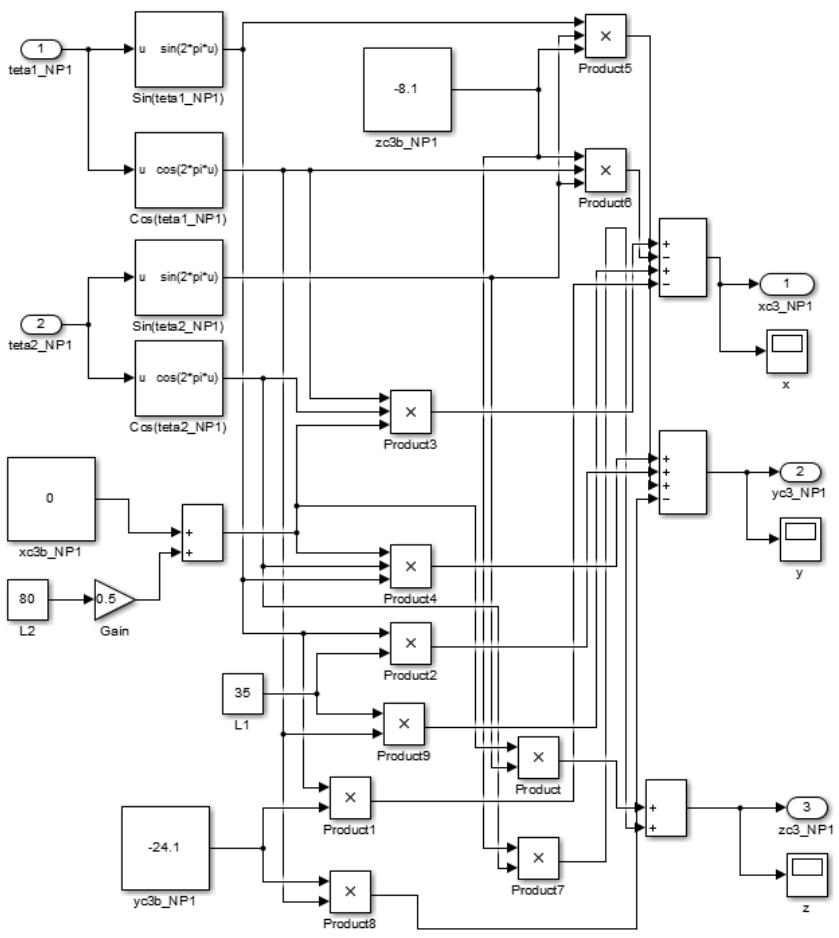

Fig. 4. Fragment of the simulation model of the position of centre of gravity of the thigh segment of the robot's leg NP1

\subsection{Results}

The first posture of the robot that was analysed is the basic configuration, where all six legs were in the support phase. The objective of the test was to verify if the model is correct and to determine the position of the robot's centre of gravity in the basic configuration. Input data that we assumed in the simulation are presented in Table 3 and the obtained results are presented in Figure 5. In this configuration all the coordinates $x$ of the position of the robot's legs located on one side should be the same. However, the differences can be noticed. Values for middle and rear legs are negligible whereas the difference between the middle and the front leg is approximately $3 \%$. It could be the result of a larger number of trigonometric functions in the model of the front body segment, which introduces the rounding error to result calculations. Differences in values of opposite legs are the result of the geometry of the segment $\mathrm{L}_{3}$ which is tilted at a $30^{\circ}$ angle. It follows that by using the middle value of the servomotor's range we obtain in case of the right legs a tilt to the outside and in case of the left legs a tilt to the inside.

Table 3. Values of generalized coordinates for the statically stable HEXAPOD configuration supported on all legs

\begin{tabular}{|c|c|c|c|c|c|c|c|c|c|c|c|}
\hline \multicolumn{2}{|c|}{$\mathbf{N P 1}$} & \multicolumn{2}{|c|}{ NP2 } & \multicolumn{2}{c|}{ NP3 } & \multicolumn{2}{c|}{ NL1 } & \multicolumn{2}{c|}{ NL2 } & \multicolumn{2}{|c|}{ NL3 } \\
\hline$\theta_{1}$ & $0^{\circ}$ & $\theta_{1}$ & $0^{\circ}$ & $\theta_{1}$ & $0^{\circ}$ & $\theta_{1}$ & $-180^{\circ}$ & $\theta_{1}$ & $-180^{\circ}$ & $\theta_{1}$ & $-180^{\circ}$ \\
\hline$\theta_{2}$ & $0^{\circ}$ & $\theta_{2}$ & $0^{\circ}$ & $\theta_{2}$ & $0^{\circ}$ & $\theta_{2}$ & $0^{\circ}$ & $\theta_{2}$ & $0^{\circ}$ & $\theta_{2}$ & $0^{\circ}$ \\
\hline$\theta_{3}$ & $-90^{\circ}$ & $\theta_{3}$ & $-90^{\circ}$ & $\theta_{3}$ & $-90^{\circ}$ & $\theta_{3}$ & $90^{\circ}$ & $\theta_{3}$ & $90^{\circ}$ & $\theta_{3}$ & $90^{\circ}$ \\
\hline
\end{tabular}




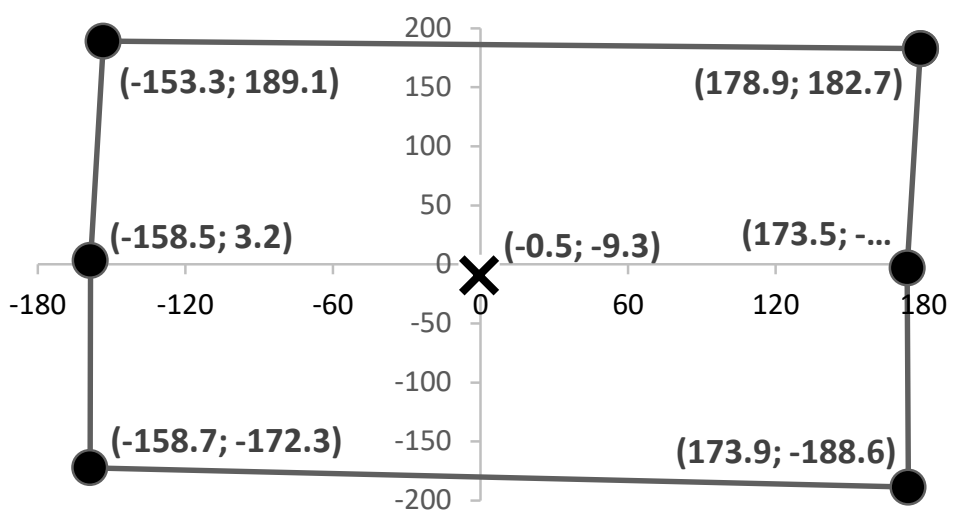

Fig. 5. Position of centre of gravity and the support polygon for the robot standing on six legs

The next two tested postures concern three-point support gait in the basic HEXAPOD configuration. Figure 6 presents the results of research performed on the basis of data from Table 4. The robot is supported on legs NP1, NP3 and NL2, and is moving legs NL1, NL3 and NP2. It can be observed that this type of gait is statically stable and has a large stability margin. In this case this parameter equals $84.3 \mathrm{~mm}$ (LSM) and $74.4 \mathrm{~mm}$ (SSM).

Table 4. Values of generalized coordinates for statically stable HEXAPOD configuration supported on legs NP1, NP3 and NL2

\begin{tabular}{|c|c|c|c|c|c|c|c|c|c|c|c|}
\hline \multicolumn{2}{|c|}{ NP1 } & \multicolumn{2}{|c|}{ NP2 } & \multicolumn{2}{c|}{ NP3 } & \multicolumn{2}{c|}{ NL1 } & \multicolumn{2}{|c|}{ NL2 } & \multicolumn{2}{c|}{ NL3 } \\
\hline$\theta_{1}$ & $0^{\circ}$ & $\theta_{1}$ & $45^{\circ}$ & $\theta_{1}$ & $0^{\circ}$ & $\theta_{1}$ & $-225^{\circ}$ & $\theta_{1}$ & $-180^{\circ}$ & $\theta_{1}$ & $-225^{\circ}$ \\
\hline$\theta_{2}$ & $0^{\circ}$ & $\theta_{2}$ & $45^{\circ}$ & $\theta_{2}$ & $0^{\circ}$ & $\theta_{2}$ & $-45^{\circ}$ & $\theta_{2}$ & $0^{\circ}$ & $\theta_{2}$ & $-45^{\circ}$ \\
\hline$\theta_{3}$ & $-90^{\circ}$ & $\theta_{3}$ & $-60^{\circ}$ & $\theta_{3}$ & $-90^{\circ}$ & $\theta_{3}$ & $60^{\circ}$ & $\theta_{3}$ & $90^{\circ}$ & $\theta_{3}$ & $90^{\circ}$ \\
\hline
\end{tabular}

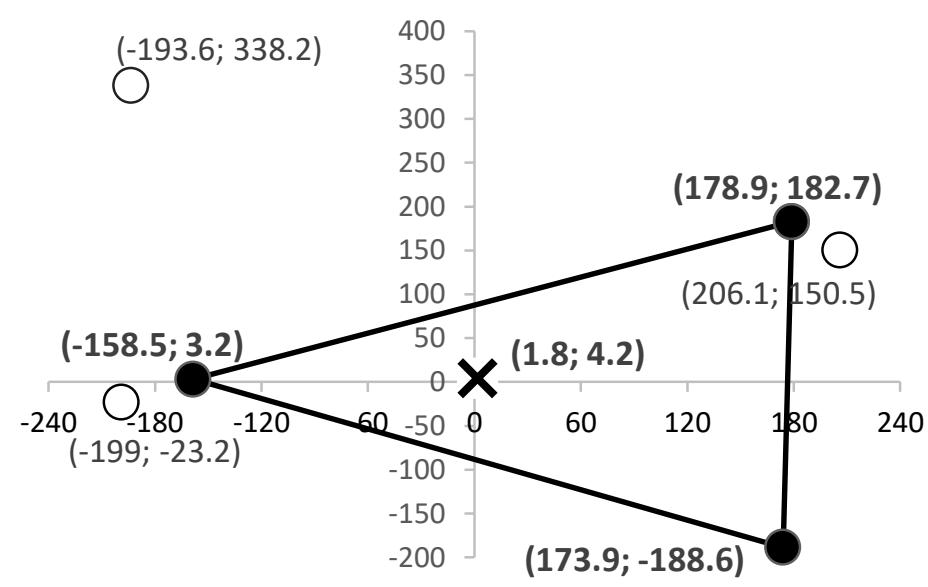

Fig. 6. Position of centre of gravity and the support polygon for the robot standing on three legs (NP1, NP3 and NL2)

In order to destabilize the robot another analysis has been conducted with the robot supported on two rear legs and the right middle leg. The data for this analysis has been included in Table 5. The analysis results for this case are presented in Figure 7. In can be clearly seen that the position of centre of gravity is located more than $90 \mathrm{~mm}$ away from the edge of the support polygon on the axis with the direction consistent with the velocity vector. It can be stated with nearly $100 \%$ certainty that in this case the robot will fall over as a result 
of losing stability. By trying to modify the position of legs and maximally extending the counterweight merely one configuration (Table 6) can be found that borders stability (Fig. 8) whose stability margin is only $0.5 \mathrm{~mm}$. It follows that for the tested design static stability of the robot can be nearly always achieved by appropriate configuration of the legs and the extendable weight. However, this is an inefficient measure and this support case should be avoided.

Table 5. Values of generalized coordinates for statically unstable HEXAPOD configuration supported on legs NP2, NP3 and NL3

\begin{tabular}{|c|c|c|c|c|c|c|c|c|c|c|c|}
\hline \multicolumn{2}{|c|}{$\mathbf{N P 1}$} & \multicolumn{2}{|c|}{$\mathbf{N P 2}$} & \multicolumn{2}{c|}{ NP3 } & \multicolumn{2}{c|}{ NL1 } & \multicolumn{2}{c|}{ NL2 } & \multicolumn{2}{|c|}{ NL3 } \\
\hline$\theta_{1}$ & $45^{\circ}$ & $\theta_{1}$ & $0^{\circ}$ & $\theta_{1}$ & $0^{\circ}$ & $\theta_{1}$ & $-225^{\circ}$ & $\theta_{1}$ & $-225^{\circ}$ & $\theta_{1}$ & $-180^{\circ}$ \\
\hline$\theta_{2}$ & $45^{\circ}$ & $\theta_{2}$ & $0^{\circ}$ & $\theta_{2}$ & $0^{\circ}$ & $\theta_{2}$ & $-45^{\circ}$ & $\theta_{2}$ & $-45^{\circ}$ & $\theta_{2}$ & $0^{\circ}$ \\
\hline$\theta_{3}$ & $-60^{\circ}$ & $\theta_{3}$ & $-90^{\circ}$ & $\theta_{3}$ & $-90^{\circ}$ & $\theta_{3}$ & $60^{\circ}$ & $\theta_{3}$ & $60^{\circ}$ & $\theta_{3}$ & $90^{\circ}$ \\
\hline
\end{tabular}

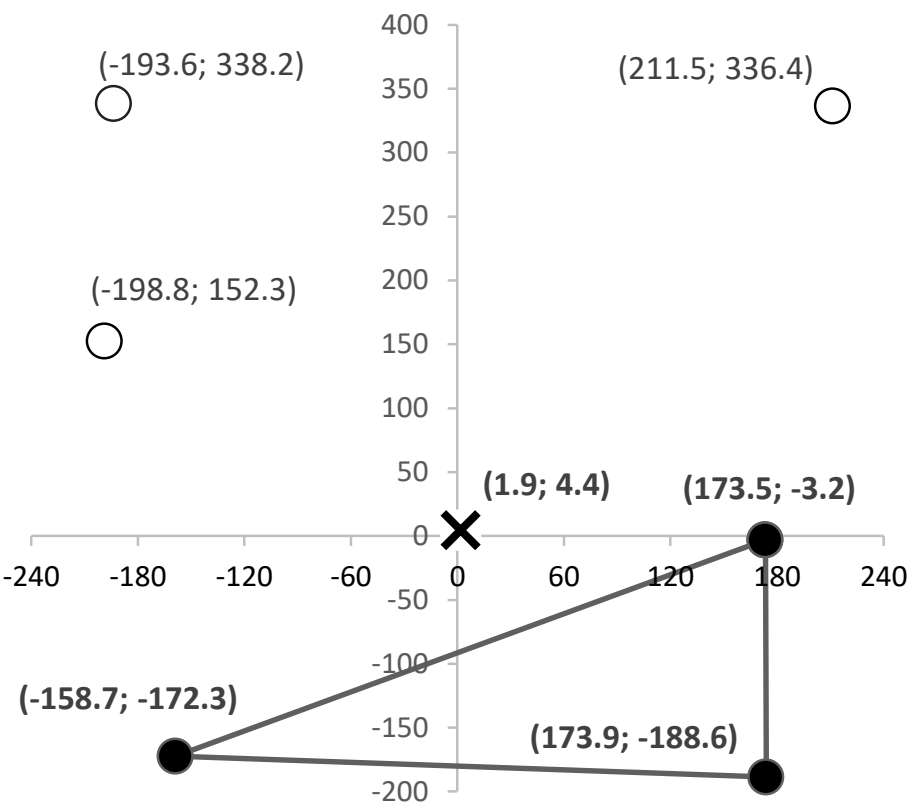

Fig. 7. Position of centre of gravity and the support polygon for the robot standing on three legs (NP2, NP3 and NL3) - lack of static stability

Table 6. Values of generalized coordinates for statically stable HEXAPOD configuration supported on legs NP2, NP3 and NL3

\begin{tabular}{|c|c|c|c|c|c|c|c|c|c|c|c|}
\hline \multicolumn{2}{|c|}{$\mathbf{N P 1}$} & \multicolumn{2}{|c|}{ NP2 } & \multicolumn{2}{|c|}{ NP3 } & \multicolumn{2}{|c|}{ NL1 } & \multicolumn{2}{|c|}{ NL2 } & \multicolumn{2}{|c|}{ NL3 } \\
\hline$\theta_{1}$ & $45^{\circ}$ & $\theta_{1}$ & $45^{\circ}$ & $\theta_{1}$ & $0^{\circ}$ & $\theta_{1}$ & $-225^{\circ}$ & $\theta_{1}$ & $-225^{\circ}$ & $\theta_{1}$ & $-225^{\circ}$ \\
\hline$\theta_{2}$ & $45^{\circ}$ & $\theta_{2}$ & $0^{\circ}$ & $\theta_{2}$ & $0^{\circ}$ & $\theta_{2}$ & $-45^{\circ}$ & $\theta_{2}$ & $-45^{\circ}$ & $\theta_{2}$ & $0^{\circ}$ \\
\hline$\theta_{3}$ & $-30^{\circ}$ & $\theta_{3}$ & $-90^{\circ}$ & $\theta_{3}$ & $-90^{\circ}$ & $\theta_{3}$ & $30^{\circ}$ & $\theta_{3}$ & $30^{\circ}$ & $\theta_{3}$ & $90^{\circ}$ \\
\hline
\end{tabular}




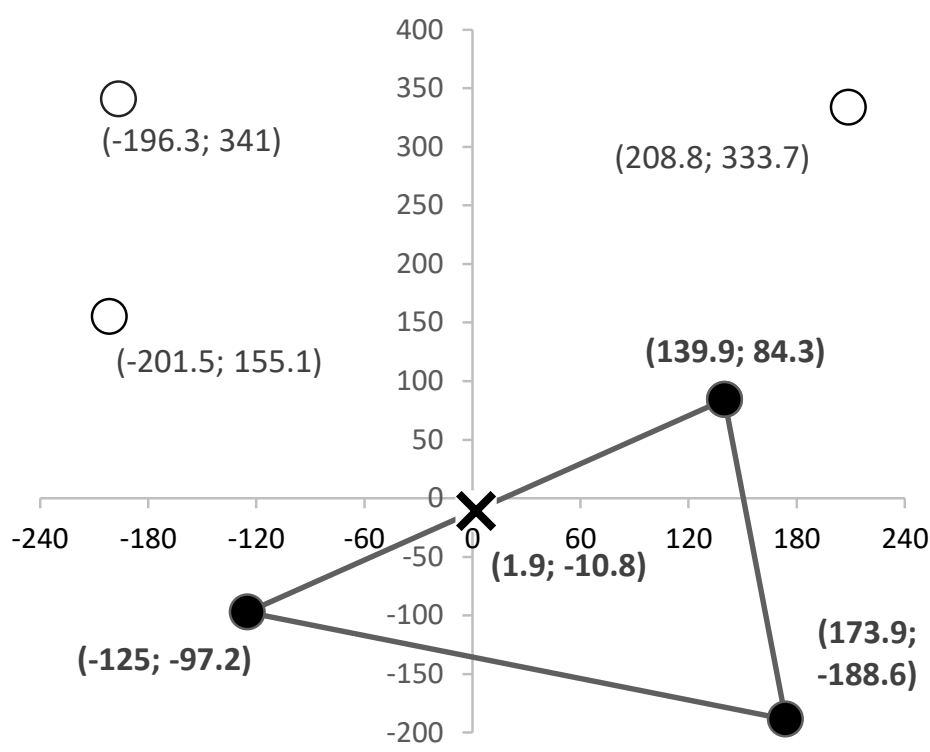

Fig. 8. Position of centre of gravity and the support polygon for the robot standing on three legs (NP2, NP3 and NL3) - achieving static stability

Further analyses concern the alternative QUADRUPED configuration in which the front body segment is elevated to $90^{\circ}$ angle and the robot moves on only two pairs of legs: the rear ones and the middle ones. In all the presented analyses the robot moves by means of the gait called crawling, which involves moving only one leg NL2 at a time. Other legs are in the retraction phase. In the first analysed example the left leg is in the neutral position whereas right legs NP2 and NP3 are extended by $45^{\circ}$ respectively to the front and to the back (Table 7). Additionally the counterweight $283.5 \mathrm{~g}$ is maximally extended to $40 \mathrm{~mm}$. Results of this analysis are presented in Figure 9.

Table 7. Values of generalized coordinates for statically unstable QUADRUPED configuration

\begin{tabular}{|c|c|c|c|c|c|c|c|c|c|c|c|}
\hline \multicolumn{2}{|c|}{ NP1 } & \multicolumn{2}{|c|}{ NP2 } & \multicolumn{2}{c|}{ NP3 } & \multicolumn{2}{|c|}{ NL1 } & \multicolumn{2}{|c|}{ NL2 } & \multicolumn{2}{|c|}{ NL3 } \\
\hline$\theta_{1}$ & $0^{\circ}$ & $\theta_{1}$ & $45^{\circ}$ & $\theta_{1}$ & $-45^{\circ}$ & $\theta_{1}$ & $-180^{\circ}$ & $\theta_{1}$ & $-225^{\circ}$ & $\theta_{1}$ & $-180^{\circ}$ \\
\hline$\theta_{2}$ & $0^{\circ}$ & $\theta_{2}$ & $0^{\circ}$ & $\theta_{2}$ & $0^{\circ}$ & $\theta_{2}$ & $-45^{\circ}$ & $\theta_{2}$ & $-45^{\circ}$ & $\theta_{2}$ & $0^{\circ}$ \\
\hline$\theta_{3}$ & $-180^{\circ}$ & $\theta_{3}$ & $-60^{\circ}$ & $\theta_{3}$ & $-60^{\circ}$ & $\theta_{3}$ & $180^{\circ}$ & $\theta_{3}$ & $30^{\circ}$ & $\theta_{3}$ & $60^{\circ}$ \\
\hline
\end{tabular}

It can be noted that despite the full extension of the counterweight centre of gravity is still located outside the area limited by the support polygon. It means that this alignment of legs is not beneficial for this configuration. The solution might be increasing counterweight or modification of angular position of the legs.

In the first attempt the left leg has been shifted $30^{\circ}$ to the front leaving other parameters unchanged (Table 8). The obtained results have been presented in Figure 10. This measure improved stability whose margins LSM and SSM are respectively $23.3 \mathrm{~mm}$ and $20.3 \mathrm{~mm}$. It can then be concluded that in terms of static stability of the hexa-quad bimorph walking robot in QUADRUPED configuration the key role is played by adequate design of algorithms generating its gait. In order to prove the presented thesis an identical simulation has been performed with the difference being that this time the counterweight is not extended. The results of this test are presented in Figure 11. 


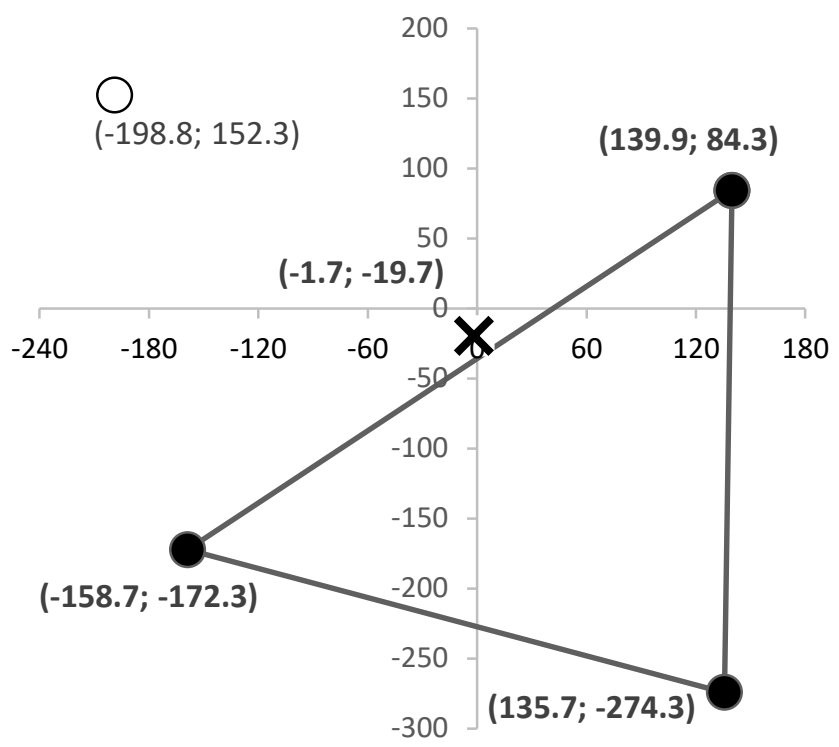

Fig. 9. Position of centre of gravity and the support polygon for the robot standing on three legs (NP2, NP3 and NL3) in the QUADRUPED configuration - lack of static stability

Table 8. Values of generalized coordinates for statically stable QUADRUPED configuration

\begin{tabular}{|c|c|c|c|c|c|c|c|c|c|c|c|}
\hline \multicolumn{2}{|c|}{ NP1 } & \multicolumn{2}{|c|}{ NP2 } & \multicolumn{2}{c|}{ NP3 } & \multicolumn{2}{|c|}{ NL1 } & \multicolumn{2}{|c|}{ NL2 } & \multicolumn{2}{|c|}{ NL3 } \\
\hline$\theta_{1}$ & $0^{\circ}$ & $\theta_{1}$ & $45^{\circ}$ & $\theta_{1}$ & $-45^{\circ}$ & $\theta_{1}$ & $-180^{\circ}$ & $\theta_{1}$ & $-225^{\circ}$ & $\theta_{1}$ & $-210^{\circ}$ \\
\hline$\theta_{2}$ & $0^{\circ}$ & $\theta_{2}$ & $0^{\circ}$ & $\theta_{2}$ & $0^{\circ}$ & $\theta_{2}$ & $-0^{\circ}$ & $\theta_{2}$ & $-45^{\circ}$ & $\theta_{2}$ & $0^{\circ}$ \\
\hline$\theta_{3}$ & $-180^{\circ}$ & $\theta_{3}$ & $-60^{\circ}$ & $\theta_{3}$ & $-60^{\circ}$ & $\theta_{3}$ & $180^{\circ}$ & $\theta_{3}$ & $30^{\circ}$ & $\theta_{3}$ & $60^{\circ}$ \\
\hline
\end{tabular}

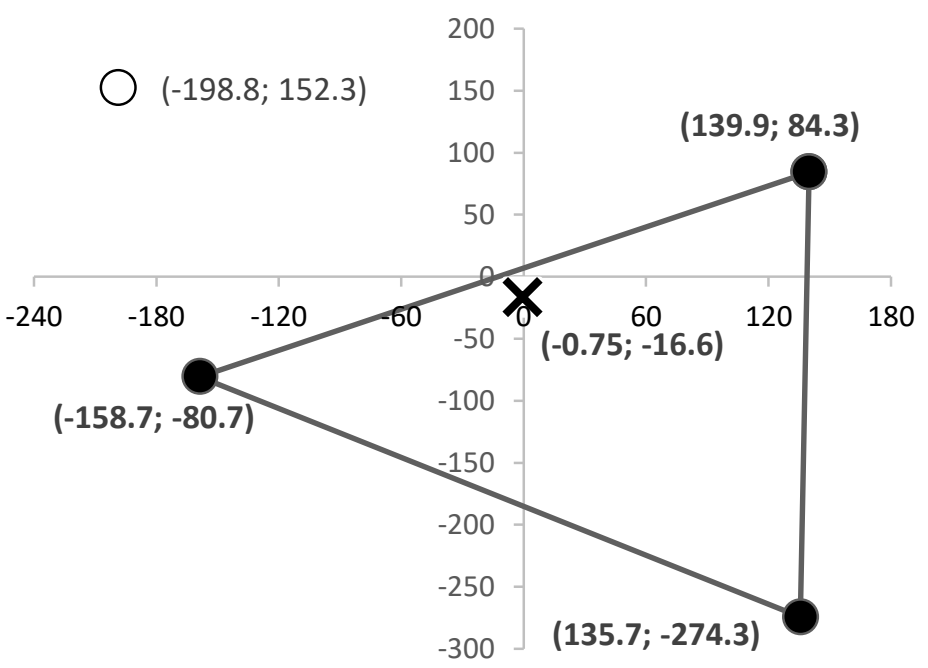

Fig. 10. Position of centre of gravity and the support polygon for the robot standing on three legs (NP2, NP3 and NL3) in the QUADRUPED configuration - achieving static stability through weight extension and adequate alignment of legs

Despite full counterweight retraction the walking robot managed to retain static stability. Stability margins for this posture are $19.5 \mathrm{~mm}$ (LSM) and $17 \mathrm{~mm}$ (SSM). The difference then 
is approximately $16 \%$. In order to determine the impact of extendable weight the last analysis has been performed, which involved increasing weight to $500 \mathrm{~g}$ resulting in an increase of approximately $75 \%$. The results obtained during this attempt are presented in Figure 12 . Obtained stability margins are $40.5 \mathrm{~mm}$ (LSM) and $35.4 \mathrm{~mm}$ (SSM). The increase in stability is comparable to the increase in weight and amounts to $74 \%$. This indicates an approximately linear correlation between the increase in weight and the growth of stability margin.

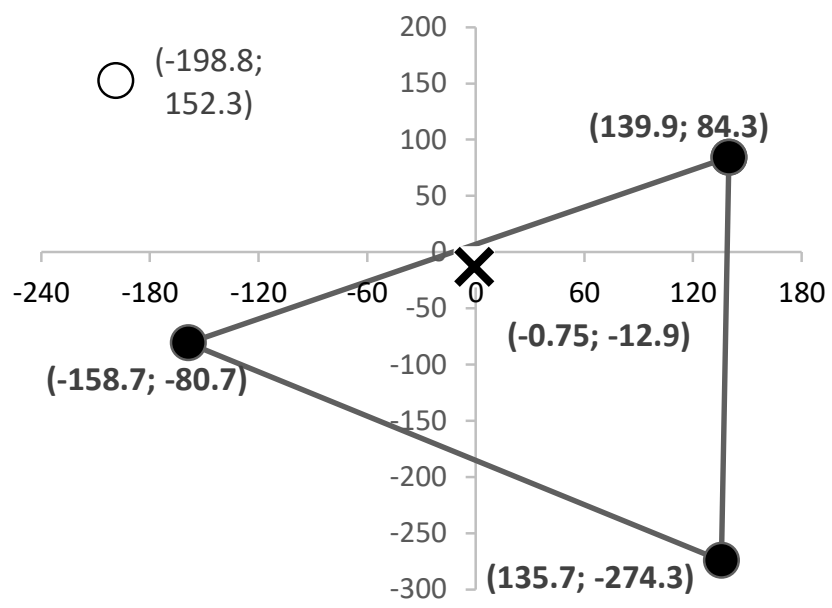

Fig. 11. Position of centre of gravity and the support polygon for the robot standing on three legs (NP2, NP3 and NL3) in the QUADRUPED configuration - adequately aligned legs without extended counterweight

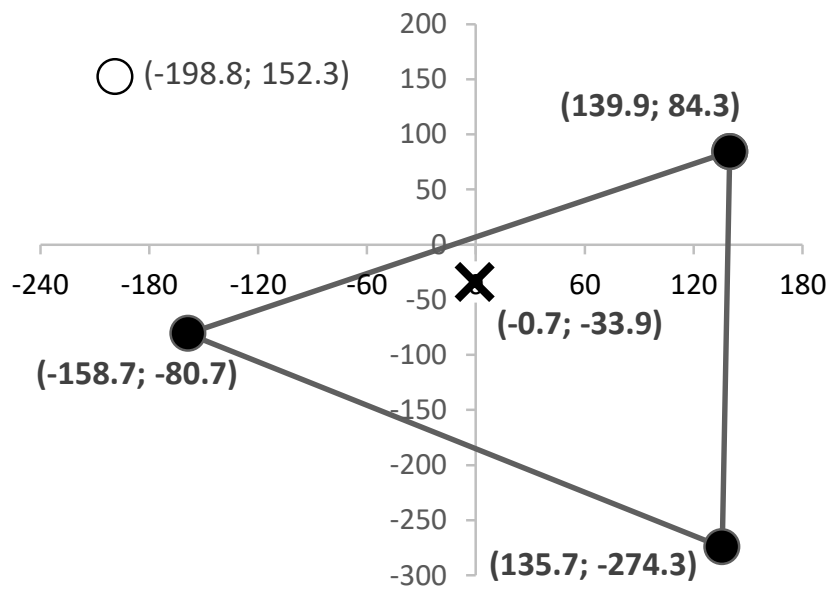

Fig. 12. Position of centre of gravity and the support polygon for the robot standing on three legs (NP2, NP3 and NL3) in the QUADRUPED configuration - adequately aligned legs with extended increased counterweight

\section{Summary}

The analyses presented in this article enabled initial verification of the assumed mathematical and simulation model. The possibilities and limitations that follow from the assumed design have been established. This concerns particularly the simulations conducted in order to 
determine the position of the robot's centre of gravity. Its position does not differ significantly from the assumed effective position which overlaps with the start of the base coordinate system.

The initial analyses demonstrated that the robot's design should enable a large range of stability during uphill movement whereas in the downhill phase it will be necessary to use more complex steering algorithms. The important conclusion is also that adequate alignment of the robot's legs ensures retention of its static stability in nearly every posture.

The presented results also made it possible to identify the robot's characteristics that need to be taken into consideration in further research. As a result, the next research stage will be extending the simulation model by the influence of surface tilt and weight of the mass being elevated. Comparative analysis of the prototype's stability with the simulation model will also be necessary. In supplementary tests, the analysis of the influence of mass distribution on the robot's gait stability will be performed. The next stage will be work related to formulation of steering and gait generation algorithms of the hexa-quad bimorph walking robot.

\section{References}

1. D. Wojtkowiak, K. Talaśka, I. Malujda, Concept of the hexa-quad bimorph walking robot and the design of its prototype. Acta Mechanica et Automatica 12, 1, 60-65, (2018)

2. M.M.A. Hajiabadi, Analytical Workspace, Kinematics, and Foot Force Based Stability of Hexapod Walking Robots [dissertation]. Worcester Polytechnic Institute (2013)

3. K. Inagaki, A gait study for one-leg-disabled hexapod robot. Adv. Robot. 12, 593-604 (1998)

4. M-H. Hung, F-T. Cheng, H-L. Lee, D.E. Orin, Increasing the stability margin of multilegged vehicles through body sway. J Chin. Inst. Eng. 28, 39-54 (2005)

5. E. Garcia, J. Estremera, P. Gonzalez-de-Santos, A comparative study of stability margins for walking machines. Robotica 20, 595-606 (2002)

6. J-Y. Kim, B-H. Jun. Design of six-legged walking robot, Little Crabster for underwater walking and operation. Adv. Robot. 28, 77-89 (2014)

7. W.A. Lewinger, M.S. Branicky., R.D. Quinn, Insect-inspired, actively compliant hexapod capable of object manipulation. In: CLAWAR Proceedings, London (UK), p. 65 - 72. (2005)

8. Y. Tang, S. Ma, Y. Sun, D. Ge, Planar legged walking of passive-spine hexapod robot. Adv. Robot. 29, 1510-1525 (2015)

9. S. Bartsch, Development, control, and empirical evaluation of the six-legged robot SpaceClimber designed for extraterrestrial crater exploration [dissertation]. University of Bremen (2012)

10. M. Manz, S. Bartsch, F. Kirchner, MANTIS - a robot with advanced locomotion and manipulation abilities. In: Symposium on Advanced Space Technologies in Robotics and Automation Proceedings, Noordwijk the Netherlands (2013)

11. D. Wojtkowiak, I. Malujda, K. Talaśka, et al., Influence of the Body Weight Distribution on the Walking Robot's Gait Stability. Proceedia Engineering 177, 419-424, (2017)

12. D. Wojtkowiak, K. Talaśka, Metodyka wyznaczania położenia środka ciężkości na przykładzie sześcionożnego bimorficznego robota kroczacego. In: Nasze Stulecie Nauka dla Obronności 2018 Proceedings, Poznań, Poland (2018) 